\title{
DESARROLLO EMBRIONARIO DE LA PINTARROJA COMUN SCHROEDERICHTHYS CHILENSIS (GUICHENOT, 1848) (CHONDRICHTHYES: SCYLIORHINIDAE)
}

\author{
EMBRIONARY DEVELOPMENT OF THE REDSPOTTED CATSHARK \\ SCHROEDERICHTHYS CHILENSIS (GUICHENOT, 1848) (CHONDRICHTHYES: \\ SCYLIORHINIDAE)
}

\author{
Sebastián Hernández ${ }^{1}$, Julio Lamilla² ${ }^{2}$ Enrique Dupré1, Wolfgang Stotz ${ }^{1}$ \\ ${ }^{1}$ Facultad de Ciencias del Mar. Universidad Católica del Norte. Casilla 117, Coquimbo, Chile. \\ Email: pintarrojacl@yahoo.com \\ ${ }^{2}$ Facultad de Ciencias. Universidad Austral de Chile. Casilla 567, Valdivia, Chile.
}

\begin{abstract}
RESUMEN
El presente estudio describe el desarrollo embrionario de la pintarroja común Schroederichthys chilensis, a través de observaciones de la morfología externa de la cápsula, morfología del embrión y mediciones del tiempo de gestación. Se recolectaron 60 cápsulas de huevos en la localidad de Chungungo (IV Región). Además se obtuvieron dos cápsulas desovadas por una hembra mantenida en cautiverio. Las cápsulas fueron descritas basándose en mediciones morfométricas, coloración, textura y presencia de las fisuras respiratorias. Para la determinación y descripción de la morfología embrionaria se utilizaron embriones extraídos de las cápsulas recolectadas. Para la determinación del tiempo de gestación se observaron continuamente 15 cápsulas con embriones vivos, a través de una ventana transparente en la pared de cada cápsula. Las cápsulas de huevos son rectangulares y tienen dos prolongaciones anteriores llamadas "filamentos del ovisaco" y dos posteriores llamadas "zarcillos". Las cápsulas recientemente desovadas son de coloración parda o verde opaco, textura parcialmente lisa y pegajosa y las fisuras respiratorias están selladas. Sin embargo, las cápsulas con embriones en estados avanzados son de coloración café oscura opaca, textura lisa y las fisuras respiratorias están abiertas. Se establecieron 6 estados de desarrollo embrionario en base a los siguientes caracteres diagnósticos: saco vitelino sin embrión, aperturas faríngeas, filamentos branquiales, diferenciación de las aletas desde el pliegue dorsal y ventral, espinas en la cola, hilera de espinas dorsales y pigmentación. El tiempo de gestación es de 211 días a una temperatura entre $11,1-15,8^{\circ} \mathrm{C}$.
\end{abstract}

Palabras Claves: Desarrollo, embriones, cápsulas de huevos, pintarroja, Chile.

\section{ABSTRACT}

The present study describes the embrionary development of the redspotted shark, Schroederichthys chilensis, through observations of the external morphology of the eggs capsules, embryo morphology and measurement of gestation time. Sixty egg capsules were collected in the locality of Chungungo (IV Region, Chile). Two additional capsules were laid by a female kept in captivity. Eggs capsules were described based on morphometric measurements, coloration, texture and presence of respiratory fissures. For the determination and description of the embryonic morphology, embryos extracted from the collected capsules. For the determination of the gestation time 15 capsules with alive embryos were observed through a transparent window on the wall of each capsule. The eggs capsules are rectangular and they have two anterior prolongation called "filaments ovisac" and two posterior called "tendrils". The recently eggs capsules laid are brown or green opaque in coloration, partially smooth and sticky in texture and closed respiratory fissures. The eggs capsules with embryos in advanced stages are dark brown, flat texture and respiratory fissures open. Six stages of embrionary development were determined on the basis of the following diagnostic characters: viteline sac without embryo, pharyngeal clefts, gill filaments, differentiation of the fins from the dorsal and fold ventral ridge, spines on the tail, row of dorsal spines and pigmentation. The gestation time is 211 days at temperature of $11,5-15,8^{\circ} \mathrm{C}$.

KeYwords: Development, embryos, egg capsules, catshark, Chile. 


\section{INTRODUCCION}

La pintarroja Schroederichthys chilensis (Guichenot 1848), es un tiburón demersal que habita en la zona sublitoral rocosa, entre 1-50 m de profundidad (Fariña \& Ojeda 1993). Se distribuye en el Pacífico Sur Oriental desde Ancón (Perú) hasta Chiloé (sur de Chile) (Chirichigno 1974, Chirichigno \& Cornejo 2001, Compagno 1984). En Chile, esta especie no tiene importancia económica, aún cuando en la zona costera de Coquimbo, es capturada incidentalmente por la pesquería artesanal de espinel de Sebastes capensis (Gmelin 1789) y Pinguipes chilensis (Valenciennes 1833) y por pescadores aficionados a la captura de Graus nigra (Philippi 1887) desde el borde costero rocoso. En la zona sur de Chile (desde Valdivia hasta Chiloé), es capturada incidentalmente por buques arrastreros, espineles y redes de pescadores artesanales, cuya captura es destinada a la producción de harina de pescado (Lorenzen et al. 1979). Los únicos datos acerca de la biología reproductiva de $S$. chilensis, es la agregación de individuos de diferentes sexo, durante el otoño en la localidad de San Antonio (Miranda 1980), y la presencia de hembras con cápsulas de huevos en su interior, durante un ciclo anual en la zona central de Chile (Fariña \& Ojeda 1993). El objetivo del presente estudio es describir el desarrollo embrionario de $S$. chilensis, a través de la descripcion de las cápsulas de huevo, la morfología embrionaria y el tiempo de gestación durante el desarrollo.

\section{MATERIALES Y METODOS}

Las cápsulas de huevos de S. chilensis, se recolectaron el 20 de Julio del 2002, mediante buceo apnea en el submareal rocoso (10 $\mathrm{m}$ de profundidad), desde trozos de fronda y estipe de Lessonia trabeculata Villouta \& Santelices, en la localidad de Chungungo (2937'S, $\left.71^{\circ} 19^{`} \mathrm{~W}\right)$. La morfología externa de 60 cápsulas fue descrita en base a mediciones morfométricas, coloración, textura y presencia de las fisuras respiratorias. Además se utilizó un par de cápsulas desovadas por una hembra adulta mantenida en cautiverio con agua de mar microfiltrada circulante y a temperatura ambiente. Se extrajeron 60 embriones de las cápsulas descritas anteriormente, las cuales fueron observadas y descritas mediante microscopía electrónica de barrido y microscopía de luz. Al momento de obtener el embrión se desconocía el tiempo de gestación. Sin embargo, a través de su morfología se asignó un estado de desarrollo relativo. El criterio utilizado para esta asignación, está basado en las observaciones de Castro et al. (1988). Este autor se basó en las siguientes estructuras: arcos branquiales, aperturas faringeas, filamentos branquiales, esbozos y pliegues de las aletas, ojos, espiráculos y espinas. La duración total del tiempo de gestación se determinó observando el desarrollo de 15 cápsulas mantenidas en cautiverio con agua de mar microfiltrada circulante y temperatura ambiente. Para determinar el estado de desarrollo y observar el embrión contenido en cada cápsula, se extrajeron manualmente las primeras capas pigmentadas de una de las caras superficiales de la cápsula, dejando sólo una capa transparente. De esta manera, fue posible monitorear el cambio de estado y determinar el tiempo de gestación.

\section{RESULTADOS}

Las cápsulas son rectangulares (longitud 51,24 \pm 2,47 y ancho $20,6 \pm 1,18$ ) (Tabla I), tienen dos estructuras gruesas que nacen desde el borde lateral a nivel de la mitad del tercio anterior de la cápsula (una por cada lado) (Fig. 1). Estas estructuras son filamentosas y están formadas por muchas fibras entrelazadas. Las prolongaciones anteriores son llamadas "filamentos del ovisaco" y las otras, posteriores o "zarcillos". Los filamentos del ovisaco divergen en forma paralela al eje longitudinal al igua que los posteriores que son parcialmente espiralados, ambos presentan una textura similar a las paredes de la cápsula, con sus márgenes laterales fuertemente reforzados. Las cápsulas al momento de ser desovadas son de coloración parda o verde opaca y su textura es parcialmente lisa y pegajosa con las paredes bastante rígidas. En cambio, las cápsulas con embriones en estados avanzados, prontas a eclosionar, son de coloración café oscura opaca, su textura es lisa y poco pegajosa y sus paredes son menos rígidas (blandas). Durante el desarrollo embrionario, la coloración de las prolongaciones presenta la misma evolución del color que las cápsulas. En las esquinas de las cápsulas se encuentran cuatro fisuras respiratorias, 
dos anteriores y dos posteriores. Estas fisuras, en cápsulas recién desovadas no se ven a simple vista, pero a medida que transcurren los primeros estados se hacen más notorias, aun cuando permanecen cerradas por la presencia de un material gelatinoso. En embriones de desarrollo medio, este material comienza a disolverse y las fisuras se abren, permitiendo así el flujo del agua hacia el interior de la cápsula durante el resto del desarrollo.

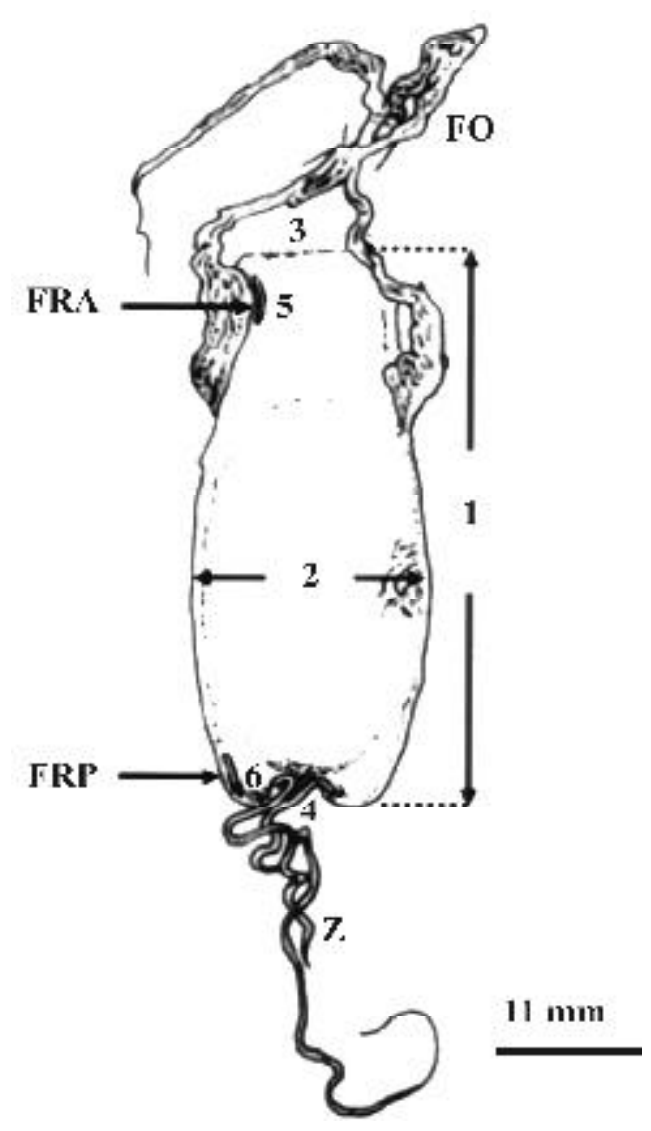

FIGURA 1. Ilustración de la cápsula de huevo, mostrando las mediciones morfométricas y la terminología adoptada. Las mediciones 1-6 están en la Tabla I; fisura respiratoria anterior (FRA); fisura respiratoria posterior (FRP); filamentos del ovisaco (FO); zarcillos (Z).

Figure 1. Illustration of egg capsule showing morphometric measurements and adopted terminology. Measurements 1-6 are in Table 1; anterior respiratory fissure (FRA); posterior respiratory fissure (FRP); filaments ovisac (FO); tendrils (Z).
De acuerdo a la aparición de estructuras morfológicas externas y cambios en la coloración, se establecieron 6 estados macroscópicos de desarrollo embrionario relativo:

Estado I Al inicio del desarrollo se observa el embrión con un saco vitelino ovalado de coloración amarillo-anaranjado y un polo animal que contiene el disco embrionario desde el cual se origina el embrión propiamente tal.

Estado II: El embrión es translúcido y está unido al saco vitelino por un ancho pedúnculo vitelino. Aparecen cuatro pares de aperturas faríngeas definidas a ambos lados de la región cefálica del embrión (Fig. 2). La primera corresponderá al futuro espiráculo o hiomandibular y la segunda a la primera hendidura branquial o hiobranquial. Ambas aperturas están completamente abiertas, sin embargo las dos restantes están comenzando a abrirse. Posteriormente en el desarrollo de este estado, aparecen y se abren las 2 últimas aperturas faríngeas, completando el set de 6 pares.

Estado III: El embrión es más alargado y robusto y está unido al saco vitelino por un delgado pedúnculo vitelino. Desde los espiráculos y las hendiduras branquiales aparecen pequeños y delgados filamentos branquiales ricamente irrigados, que comienzan a crecer durante el desarrollo de este estado (Fig. 3).

Estado IV: En este estado se diferencian los pliegues dorsal y ventral. El pliegue dorsal se extiende desde la región media hacía el extremo distal del embrión, las que formarán la primera y segunda aleta dorsal y caudal. El pliegue ventral se extiende en posición sagital, desde el esbozo de las aletas pélvicas hacía el extremo distal del embrión, las que formarán la aleta caudal y anal (Fig. 4). En el extremo distalcaudal de los pliegues dorsal y ventral se observan 5 mamelones mesodérmicos alargados ubicados en los bordes ventral y dorsal (Fig. 5A), donde posteriormente eclosionan pequeñas espinas con bordes aserrados (Fig. 5B y 6). En las regiones laterales se observan mamelones de menor tamaño que los anteriores y que originan espinas más pequeñas. 
Estado V: En la región antero-dorsal del tronco del embrión se observan dos hileras de mamelones mesodérmicos. Cada mamelon presenta una pequeña espina, que posteriormente eclosiona, formando dos hileras de espinas (Fig. 7). El embrión adopta la morfología final del desarrollo y comienza la pigmentación dorsal con manchas café oscuro y negro desde los extremos del eje del cuerpo del embrión.

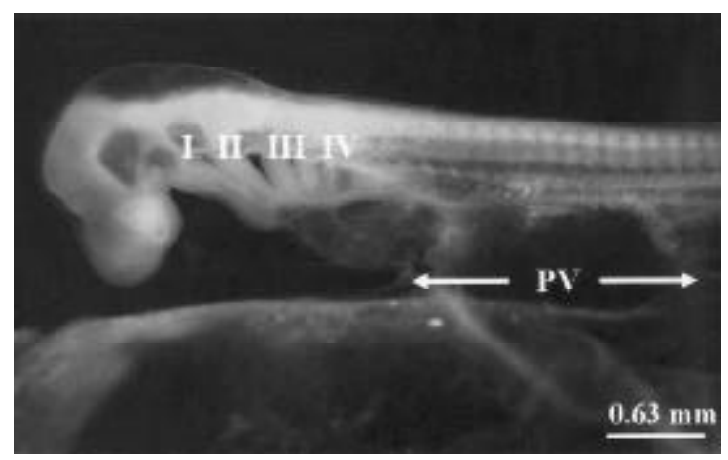

FIgURA 2. Vista lateral anterior del embrión en estado II, mostrando cuatro aperturas faríngeas (números romano) y el ancho pedúnculo vitelino (PV).

Figure 2. Anterior lateral view of embryo in stage II showing four pharyngeal clefts (Roman numeral) and width yolk stalk (PV).

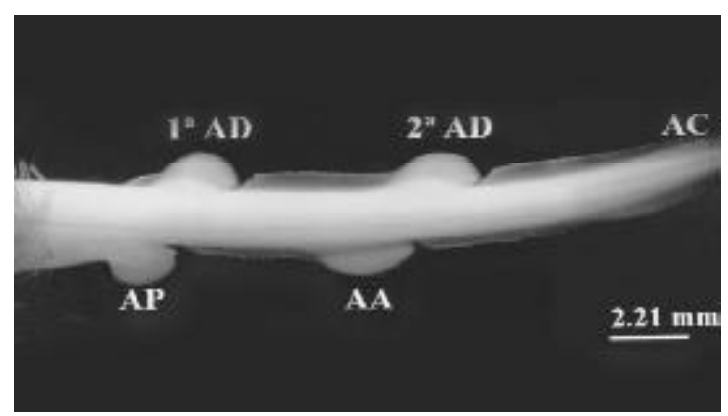

Figura 4. Vista lateral del tronco y cola del embrión en estado IV, mostrando la $1^{\mathrm{a}}$ aleta dorsal $\left(1^{\mathrm{a}} \mathrm{AD}\right) ; 2^{\mathrm{a}}$ aleta dorsal ( $\left.2^{\mathrm{a}} \mathrm{AD}\right)$; aleta pélvica (AP); aleta anal (AA); aleta caudal (AC).

FiguRe 4. Lateral view of trunk and tail of embryo in stage IV showing the $1^{\text {st }}$ dorsal fin $\left(1^{\mathrm{a}} \mathrm{AD}\right), 2^{\text {nd }}$ dorsal fin $\left(2^{\mathrm{a}}\right.$ $\mathrm{AD}$ ); pelvic fin (AP), anal fin (AA); caudal fin (AC).
Estado VI: El embrión esta completamente pigmentado con grandes manchas de coloración negro de formas irregulares y su cola esta flexionada en $180^{\circ}$ en el interior de la cápsula. El pequeño saco vitelino es de coloración amarillo claro y esta prácticamente vació (Fig. 8).

El tiempo de gestación promedio del desarrollo embrionario fue de 211 días (DE 9,1 días, $\mathrm{n}=15$ ), a temperatura ambiente $\left(11,1-15,8^{\circ} \mathrm{C}\right)$.

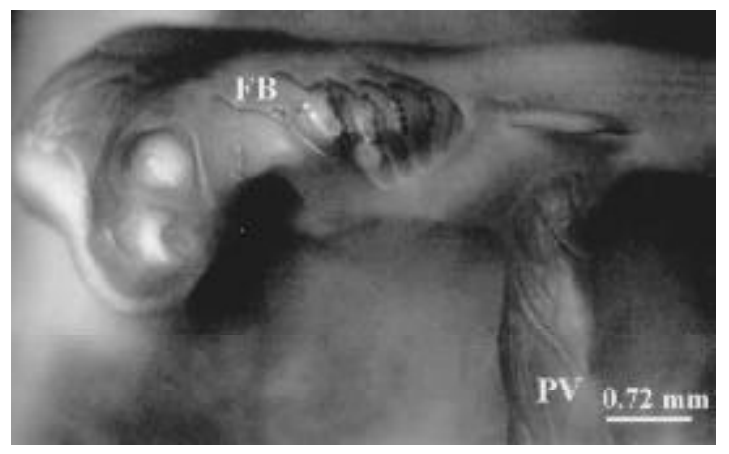

FIGURA 3. Vista lateral de la región anterior del embrión en estado III, mostrando los filamentos branquiales (FB).

FIGURE 3. Lateral view of anterior region of embryo in stage III showing the gills filaments (FB).

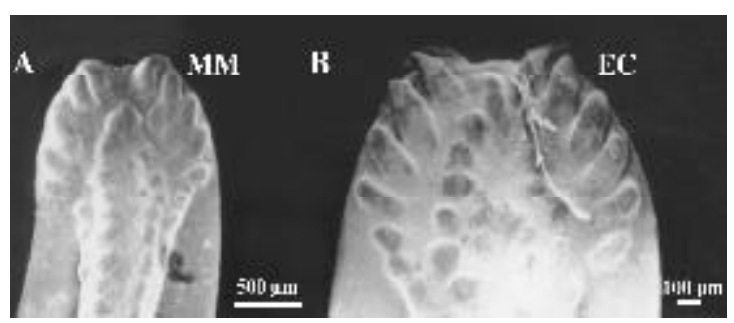

FIgURA 5. Fotografía electrónica de barrido del extremo caudal del embrión en estado IV. A mamelones mesodérmicos alargados (MM); B espinas caudales (E).

FIGURE 5. Scanning electron micrographs of caudal end of embryo in stage IV. A enlarged mesoderm thickened; B caudal spines (E). 
Desarrollo embrionario de Schroederichthys chilensis: HeRnández, S. ET AL.

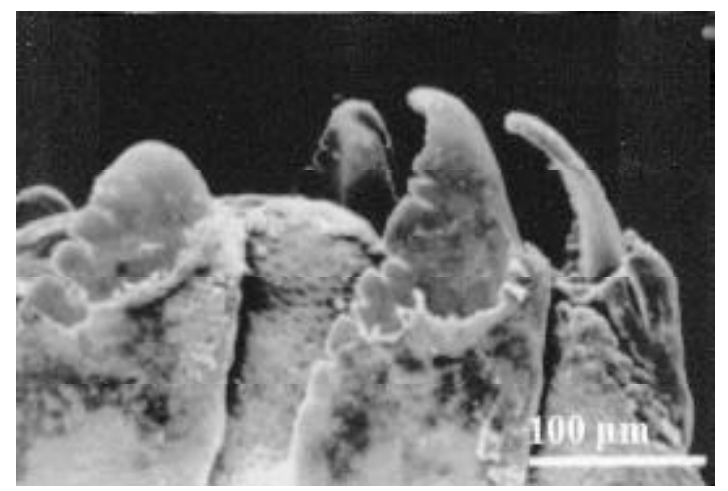

FIGURA 6. Fotografía electrónica de barrido de las espinas caudales del embrión en estado IV.

Figure 6. Scanning electron micrograph of caudal spines of embryo in stage IV.

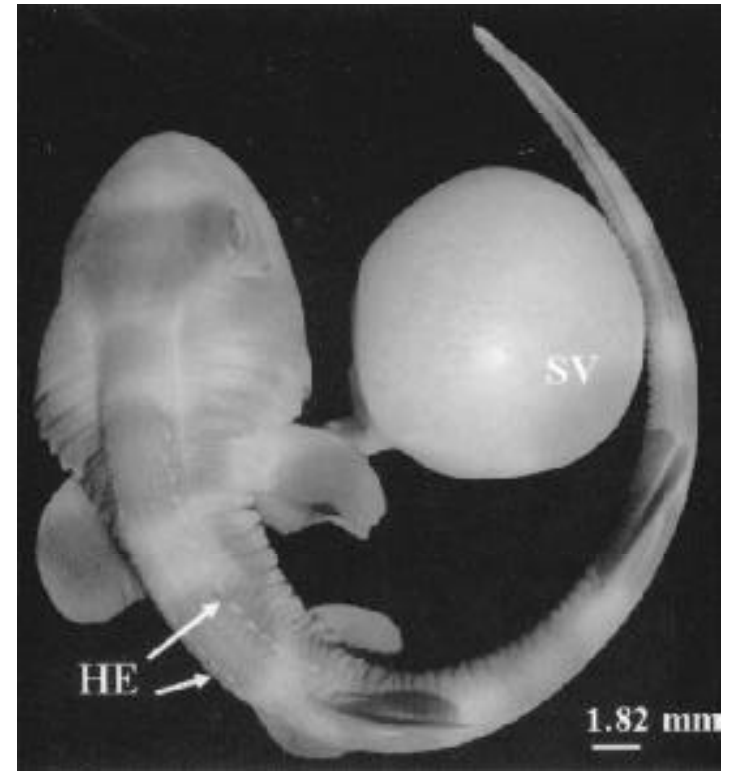

FIgURA 7. Vista dorsal del embrión en estado V, mostrando dos hilera de espinas (HE).

FIGURE 7. Lateral view of embryo in stage $\mathrm{V}$ showing two spines lines (HE)

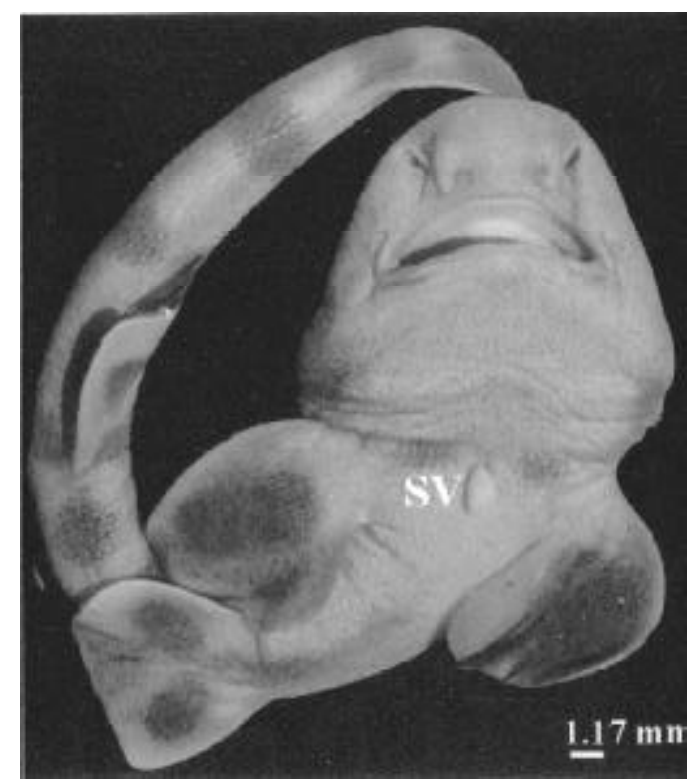

FIGURA 8. Vista ventral del embrión en estado VI, mostrando el saco vitelino (SV).

FIGURE 8 . Ventral view of embryo in stage VI showing the yolk sac (SV). 
Gayana 69(1), 2005

TABLA I. Medidas morfométricas (en mm) de las cápsulas de huevos.

TABLE I. Morphometric measurements (in $\mathrm{mm}$ ) of egg capsules.

\begin{tabular}{lccc}
\hline Medidas morfométricas & X & & DS \\
\hline 1. Longitud & 51.24 & \pm & 2.47 \\
2. Ancho & 20.60 & \pm & 1.18 \\
3. Longitud borde anterior & 11.11 & \pm & 1.01 \\
4. Longitud borde posterior & 1.72 & \pm & 1.02 \\
5. Longitud fisura respiratoria anterior & 0.93 & \pm & 0.76 \\
6. Longitud fisura respiratoria posterior & 0.79 & \pm & 0.17 \\
\hline
\end{tabular}

\section{DISCUSION}

Las cápsulas de la pintarroja $S$. chilensis han sido descritas en forma superficial por Lorenzen et al. (1979) y por Leible et al. (1981). El primer autor sólo caracteriza las cápsulas provistas de "cuatro esquinas de largos filamentos enroscados para adherirse a las algas submareales". Sin embargo, esta descripción no diferencia la morfología externa entre ambas prolongaciones (anteriores y posteriores), descritas aquí. El segundo autor en su atlas anatómico de $S$. chilensis detalla una cápsula encontrada en el ovisaco de una hembra, donde señala la existencia de las prolongaciones semianteriores, las que denomina como "filamentos del ovisaco". Actualmente no hay información en la literatura acerca de las cápsulas de Schroederichthys bivius (Müller \& Henle 1838), sin embargo una revisión personal realizada en un ejemplar adulto hembra de $S$. bivius (fijado en formol), de la sección de Ictiología del Museo Argentino de Ciencias Naturales (sin numeración), presentaba en el extremo final de cada oviducto una cápsula de similar forma y aspecto que las de $S$. chilensis. No obstante, debido a las malas condiciones en las cuales estaba preservada se destrozaron al ser manipuladas, y no se pudo realizar un análisis más fino. Junto a la cápsula de Schroederichthys tenius descrita por Gomes (1995), las cuatro especies del género Schroederichthys presentarían un patrón similar del género, respecto a la morfología externa de las cápsulas de huevo y las prolongaciones anteriores y posteriores. Las prolongaciones anteriores son medianamente largas e hilachentas y las prolongaciones posteriores son largas y notoriamente espiraladas.
El desarrollo embrionario de S. chilensis es similar al de otras especies pertenecientes al género Scyliorhinus (Castro et al. 1988; Ballardet al. 1993). No obstante, el desconocimiento del tiempo en el cual las cápsulas fueron desovadas y enredadas en el alga, fue imposible utilizar una tabla de tiempo exacta en la cual se registrase cada evento o aparición de una estructura reconocible. No obstante, a pesar de esta limitación, los estados embrionarios aquí descritos son útiles para la comparación del desarrollo embrionario, estableciendo las equivalencias entre los estados. En este contexto Ballard et al. (1993) reconoce un total de 34 estados, siendo los estados desde el 21 al 34 equivalentes a los estados 2 al 6 identificados para S. chilensis.

La ornamentación de espinas en el extremo de la cola es una característica de los embriones de Scyliorhinidos (Ballard et al. 1993). Éstas han sido reportadas para Scyliorhinus retifer (Garman 1881) (Castro et al. 1988) y detalladas para Scyliorhinus canicula (Linnaeus 1758) (Ballard et al. 1993). La ornamentación de espinas y la flexión de la cola en $180^{\circ}$ que adopta el embrión de $S$. chilensis en sus últimas semanas de desarrollo, facilitan la circulación continua del agua de mar desde las fisuras respiratorias hacia la región branquial del embrión (Luer \& Gilbert 1985, Castro et al. 1988).

La presencia de las dos hileras de espinas ubicadas en el dorso de individuos juveniles pintarrojas, para las dos especies que ocurren en nuestras costas chilenas, han sido reportadas por Norman (1937) y Petit \& Budker 1937 in Springer (1979), siendo aparentemente diferentes entre ambas especies. No obstante, según Springer (1979) estas diferencias entre las dos especies presentan dudas, ya que no 
hay certeza de que se hayan examinado juveniles de ambas especies. De darse realmente esa diferencia, la forma, disposición y número de estas espinas podrían ser de importancia taxonómica, para identificar juveniles de ambas especies de pintarrojas.

El tiempo de gestación de S. chilensis entre 11,1$15,8^{\circ} \mathrm{C}$, se estima en 211 días, siendo similar al descrito para la especie Scyliorhinus retifer (256 días \pm 8 días, $\mathrm{n}=62$ ) a temperaturas entre $11,7-12,8$ ${ }^{\circ} \mathrm{C}$ (Castro et al. 1988), y para $S$. canicula (175días) a $16^{\circ} \mathrm{C}$ (Ballard et al. 1993). La temperatura utilizada en nuestro estudio es equivalente a la temperatura superficial de bahía "La Herradura", que es una bahía muy protegida en que la temperatura de las aguas superficiales generalmente aumenta durante los meses de verano. En lugares costeros expuestos y en profundidades de $10 \mathrm{~m}$, en que normalmente se encuentran las posturas de S.chilensis, se pueden esperar temperaturas menores a las utilizadas en el experimento. Si es así, también se podría esperar que tanto $S$. chilensis como especies afines (misma familia y género), podrían presentar un período de desarrollo mas largo, eventualmente de un año como lo señala Castro et al. (1988), tema que sin embargo aun necesita mayor investigación.

\section{AGRADECIMIENTOS}

Se agradece la colaboración al Dr. Gustavo Chiaramonte del Museo Nacional Argentino "Bernardino Rivadavia" por el préstamo de los ejemplares S. bivius y por su ayuda; por el financiamiento de la tesis de la que forma parte este trabajo, al Doctor Wolfgang Stotz y al M.Sc Enrique Dupré. A la Universidad Católica del Norte por la disponibilidad de espacio para realizar esta investigación.

\section{BIBLIOGRAFIA}

Ballard, W. W., J. Mellinger \& H. Lechenault. 1993.A series of normal stages for development of Scyliorhinus canicula, the lesser spotted dogfish (Chondrichthyes: Scyliorhinidae). J. Exp. Zool. 267: 318-336.

Castro, J. I., P. M. B ubucis \& N.A. Overstrom. 1988. The reproductive biology of the chain dogfish, Scyliorhinus retifer. Copeia 1988(3): 740-746.

Chirichigno, N. 1974. Clave para identificar los peces marinos del Perú. Instituto del Mar del Perú. Callao. Informe $\mathrm{N}^{\circ}$ 44: 387pp.

Chirichigno, N. \& M. Cornejo. 2001. Catálogo comentado de los peces marinos del Perú. Instituto del Mar del Perú. Publicación especial. Callao. 314 pp.

Compagno, L. J. V. 1984. FAO species catalogue. Sharks of the World. An annoted and illustrated catalogue of shark species known to date. Part 2. Carcharhiniformes. FAO Fisheries Synopsis. No. 125. 4: 251-655.

FARIÑA, J. M. \& P. OJEDA. 1993. Abundance, activity, and trophic patterns of the redspotted catshark, Schroederichthys chilensis, on the Pacific temperate coast of Chile. Copeia 1993(2): 545-549.

Gomes, L.U. 1995. Egg capsules of Schroederichthys tenuis and Scyliorhinus haeckelii. Copeia 1995:232-233.

Leible, M., D. M. Dittus \& C. G. Belmar. 1981. Atlas anatómico de la pintarroja, Schroederichthys chilensis. Universidad Católica de Chile., Talcahuano, Chile., Vol 1: 113 pp.

Lorenzen, S., C. Gallardo., C. Jara., E. Gasing, G. Pequeño \& C. Moreno. 1979. Mariscos y peces de importancia comercial en el sur de Chile. Universidad Austral de Chile. Valdivia. $131 \mathrm{pp}$.

Luer, C. A \& P. W. Gilbert. 1985. Mating behavior, egg deposition, incubation period and hatching in the clearnose skate, Raja eglenteria. Environmental Biology of Fishes 13 (3): 161-171.

MiRANDA, O. 1980. Agregación sexual en Schroederichthys chilensis (Guichenot 1848) durante la parición. Not. Mens. Mus. Nac. Hist. Nat., Santiago 24: 7-9.

Norman, J. R. 1937. Coast fishes. Part II. The Patagonian region. Discovery Reports 16:1-150.

Springer, S. 1979. A revision of the catsharks, family Scyliorhinidae. NOAA Tech. Rep. NMFS Circ. 422: 1-97. 